\title{
Rapid Sequence Induction of Anaesthesia in the Paediatric Patient: Controversies and proposed protocol
}

\section{MR Correia}

Specialist Anaesthetist, Department of Anesthesia, Chris Hani Baragwanath Academic Hospital, University of the Witwatersrand Corresponding author, email:drmichelle.correia@gmail.com

\section{Introduction}

The best way to perform a Rapid sequence induction (RSI) of anaesthesia in the paediatric patient has been a longstanding contentious issue amongst practicing anaesthetists. Paediatric patients have many anatomical and physiological differences to adults that make the performance of a classical RSI inappropriate in these patients.

Over the last few years, the international literature has examined the actual risk of peri-operative pulmonary aspiration in children and found it to be much lower than previously thought. ${ }^{1-3}$ Studies have also shown that even once pulmonary aspiration had occurred, the associated morbidity and mortality is not significant. ${ }^{1-4}$

The reasons why children regurgitate and aspirate have recently been assessed. There is good evidence to show that regurgitation, vomiting and aspiration is caused by direct laryngoscopy being performed while the patient is at an inadequate depth of anaesthesia and muscle relaxation during induction and intubation. These complications occurred with the classical method of RSI and in some cases were directly associated with the administration of cricoid pressure. ${ }^{4-6}$ Hypoxia, hypercarbia and cardiovascular deterioration were all associated with the performance of classical RSI in children. ${ }^{7-8}$

The steps involved in a classical RSI are no longer deemed appropriate in paediatric anaesthetic practice for the following reasons:

\section{- Pre-oxygenation:}

- It is often difficult to ensure adequate pre-oxygenation of children because they are unco-operative and scared. Cessation of spontaneous or assisted ventilation in infants leads to rapid development of hypoxaemia ( $<1$ minute) without pre-oxygenation. However, even if effective pre-oxygenation is possible, time to desaturation is only marginally prolonged..$^{9}$ It is thought that the extra distress caused by forcing the child to accept the mask for the few minutes needed for pre-oxygenation is not worth the marginal benefit conferred.
- IV access:

- Inhalational induction for neonates coming for pyloromyotomy is now a generally acceptable method of induction negating the need for establishing IV access pre-induction. ${ }^{11}$ IV access is not considered an absolute must for induction of anaesthesia unless there is a concern that the child may decompensate because of inadequate resuscitation pre-operatively. Assessing the hydration status of the patient (capillary refill time, static measures of intra-vascular volume, heart rate, level of consciousness, arterial or venous blood gas and input-output chart over the last 12-24 hours) is a must to determine if the child is adequately resuscitated. If not, IV access should be established and a normal saline (N/S) fluid bolus of $20 \mathrm{ml} / \mathrm{kg}$ administered pre-induction.

- IV induction agent:

- The use of a pre-determined dose of an IV induction agent often leads to cardiovascular instability in children who may not be successfully resuscitated prior to coming to theatre for an emergency procedure. Careful titration of hypnotic drugs is now advocated.

- Muscle relaxants:

- The use of suxamethonium in children is associated with a number of deleterious effects including fatal hyperkalaemic cardiac arrest, increasing intracranial pressure and muscle pain. ${ }^{12}$ An appropriate dose of a nondepolarising muscle relaxant is preferred.

- Cricoid pressure:

- The paediatric population have anatomical and physiological differences that make managing the airway more challenging than in adults. Cricoid pressure distorts the fragile paediatric airway and increases the incidence of difficult intubation in these already challenging airway situations. Cricoid pressure has also been shown to decrease the lower oesophageal sphincter tone and cause bucking and straining in inadequately paralysed patients. ${ }^{1,7}$ This all leads to increased risk of pulmonary aspiration and cricoid pressure is no longer recommended as a part of performing a RSI in children. 
- Time to intubation and the apnoeic period:

- The apnoea tolerance in children is reduced to such an extent that even short periods of apnoea cause rapid desaturation, hypoxia, hypercapnia and possible cardiovascular deterioration. ${ }^{7-9,13}$ Waiting for the "magical minute" to secure the airway without bag mask ventilation as described with the classical RSI in adults, has been shown to be associated with severe complications ${ }^{9}$. Gentle bag mask ventilation and inflation of the lungs to pressures not greater than $12 \mathrm{cmH}_{2} \mathrm{O}$ have shown to prevent hypoxia and hypercarbia and allows time to achieve adequate muscle relaxation. This actually decreases the risk of coughing, straining and subsequent aspiration at intubation..$^{5,9}$

- Type of endotracheal tube

- The use of cuffed endotracheal tubes (ETT) in paediatrics is also cause for debate. ${ }^{14}$ Theoretically, there are a number of advantages to using a cuffed ETT including definitive airway protection, better ventilation and oxygenation, more precise monitoring of end tidal carbon dioxide intra-operatively and decreased consumption of volatile anaesthetic agents. However, cuffed ETTs seem to be different between suppliers making the size and positioning of the cuff on the ETT unreliable. This can lead to several problems including excessive pressure from the cuff being transmitted to the glottis, cuff herniation through the vocal cords compromising ventilation and damage to the tracheal mucosa from excessive inflation. All of these factors may lead to subglottic stenosis which may be difficult to treat and add significantly to the patient's overall morbidity and mortality. The make and type of cuffed ETT is therefore an important consideration and should be checked before being used.

Because of the above evidence, specialist paediatric units internationally have changed practice to doing a "controlled" $\mathrm{RSI}$ and intubation (cRSII) for their patients. After an extensive literature search, the following are key points involved in a cRSII:7,13

- Sufficient depth of anaesthesia and adequate muscle relaxation must be achieved before direct laryngoscopy and intubation are performed so as to limit the risk of aspiration and airway trauma.

- Maintenance of depth of anaesthesia can be done via titration of IV agents or inhalational agents administered during gentle bag mask ventilation.

- Prevention of hypoxia and hypercarbia is paramount and achieved via gentle bag mask ventilation with pressures less than $12 \mathrm{cmH}_{2} \mathrm{O}$.

- Cricoid pressure is not appropriate in the paediatric patient.

- An appropriately manufactured and sized cuffed ETT should be used to secure the patient's airway if available.

\section{Protocol}

\section{Pre-operatively:}

Patient assessed as being at risk for regurgitation of gastric contents and possible pulmonary aspiration:
Indications:

- $\mathrm{NPO}<4$ hours for solids, breast milk or formula

- NPO $<2$ hours for clear fluids

- Any gastro-intestinal obstruction

- Delay of gastro-intestinal passage:

- lleus

- Peritonitis

- Renal insufficiency

- Diabetes mellitus

- Acute trauma

- Severe pain

\section{General condition of child:}

- Happy, playful, active, good interaction, well-looking

- Miserable, lethargic, ill-looking

- Vital signs:

- Check HR, NIBP, $\mathrm{SpO}_{2}$, temperature, HGT, blood results from ward chart

- Address any abnormalities with appropriate standard of care (e.g.: order blood products, take into account any organ dysfunction in the selection of anaesthesia drugs)

Volume status assessment:

- Check that the child is fully resuscitated pre-induction

- Volume status assessment

$>$ Static measures:

NIBP

» Urine output

» Skin turgor, sunken eyes, sunken fontanelle

» Blood results: urea, haematocrit, sodium

$>$ Dynamic measures:

» Heart rate

» Level of consciousness

"Capillary refill time

»Arterial or venous blood gas

* $\mathrm{SvO}_{2}$ (central venous saturation)

* Lactate

* Severity of metabolic acidosis

- If child assessed as being under-resuscitated and fluid responsive, IV access must be established awake and a fluid bolus given: $20 \mathrm{ml} / \mathrm{kg}$ bolus $\mathrm{N} / \mathrm{S}$ (or colloid:Voluven or appropriate blood products) and check response. If there is a positive response to the fluid challenge, reassess the above parameters and decide if another fluid challenge is indicated. Weigh up the risks of proceeding with the induction vs the expected benefit of time taken for extra resuscitation. Discuss with the surgeon to develop management plan.

- Assess the electrolytes - is there a need for correction? Weigh up the risk of the electrolyte disturbance against the benefit of performing emergency surgery. Approach ICU for postoperative support if required.

- Degree of abdominal distention: try to localise the cause of the GIT obstruction (upper, middle or lower GIT) or other causes such as solid organomegaly. Severe abdominal distention 
increases the risk of regurgitation and interferes with pulmonary mechanics which causes ventilation problems. A head-up position during induction helps to displace the abdominal contents away from the diaphragm which may improve ventilation during pre-oxygenation and induction.

\section{Nasogastric tube:}

- In patients with GIT obstruction, a naso- or oro-gastric tube (NGT) that is placed correctly and draining well must be in situ prior to induction of anaesthesia.

- The NGT must be suctioned with the patient in various positions. The gastric volume and pressure should be reduced as much as possible prior to induction.

Pre-medication:

- Midazolam for anxiolysis and opioids for pain control can be slowly titrated to effect.

- Caution must be used especially if the child appears clinically under-resuscitated.

- Pro-kinetics and agents that reduce gastric $\mathrm{pH}$ and volume can be used but are not routinely recommended especially in the setting of GIT obstruction.

\section{Equipment check:}

- Large bore Yankuer or soft suction catheter with strong suction vacuum must be readily available, switched on and placed at the patient's head.

- Age and weight appropriate intubation equipment

- Laryngoscope handle with bright light in good working condition

- Different size Miller and Macintosh laryngoscope blades

- Malleable introducer or intubating stylet

- Magill's forceps

- Humidification filter on circuit

- Cuffed ETTs

$>$ Equation for patient's older than 1 year: Age/4 + 3.5

$>$ Have a $0.5 \mathrm{~mm}$ internal diameter smaller size ETT available

> If no appropriately sized cuffed ETT is available, use an uncuffed ETT

- Make sure the operating table is functional and can be quickly placed in the head down position if required.

- Ensure that your anaesthetic machine is checked and that the APL valve is in good working condition.

- Have a Jackson-Reese circuit available if the child is $<20 \mathrm{~kg}$.

- Set ventilator pressure support mode with pressures limited to $<12 \mathrm{cmH}_{2} \mathrm{O}$ for gentle bag mask ventilation during induction.

\section{Patient preparation:}

- Check IV line is running well.

- Continue to treat pain and anxiety if appropriate with small doses of midazolam and opioids.

- If a NGT is in situ, place on continuous suction and leave in situ.
- Establish standard ASA monitoring

- 3 lead ECG

- Non-invasive Blood Pressure (NIBP)

- $\mathrm{SpO}_{2}$

- A neuromuscular transmission (NMT) monitor should be used if available to ensure adequate muscle relaxation prior to intubation.

- If the patient is older than 2 years of age, place in a $20^{\circ}$ headup or Anti-Trendelenburg position.

- Pre-oxygenate with $100 \% \mathrm{O}_{2}$ and a tight fitting mask if possible

- Institute oxygen analysis and $\mathrm{ETCO}_{2}$ monitoring

\section{Induction:}

Opioids such as fentanyl $1-3 \mathrm{mics} / \mathrm{kg}$ or sufentanil $0.1-0.3 \mathrm{mics} / \mathrm{kg}$ can be titrated in slowly prior to induction.

For patients in cardiovascular shock:

- Induction agent

- Ketamine $2 \mathrm{mg} / \mathrm{kg}$

- Etomidate $0.3 \mathrm{mg} / \mathrm{kg}$

- Muscle relaxant

- Suxamethonium 1-2mg/kg

- Atracurium $1 \mathrm{mg} / \mathrm{kg}$

- Cisatracurium $0.15 \mathrm{mg} / \mathrm{kg}$

- Rocuronium $1 \mathrm{mg} / \mathrm{kg}$ (for longer procedures and post-op ventilation)

For neonates and infants $<3$ months who are haemodynamically stable:

- Induction agent

- Propofol 4-5mg/kg titrated in slowly

- Muscle relaxant

- Atracurium $1 \mathrm{mg} / \mathrm{kg}$

- Cisatracurium $0.15 \mathrm{mg} / \mathrm{kg}$

For infants and children $>3$ months who are haemodynamically stable:

- Induction agent

- Propofol 3-4mg/kg titrated in slowly

- Muscle relaxant

- Atracurium $1 \mathrm{mg} / \mathrm{kg}$

- Cisatracurium $0.15 \mathrm{mg} / \mathrm{kg}$

\section{NO CRICOID PRESSURE}

\section{Ventilation via facemask:}

- Gentle bag mask or ventilator driven (on pressure support mode) ventilation is performed to prevent the rapid desaturation and hypoxia associated with apnoea in children

- Peak inspiratory pressures (PIP) must not exceed $12 \mathrm{cmH}_{2} \mathrm{O}$

- High frequency, low pressure and low volume hand ventilation with a Jackson-Reese circuit may also be used to prevent the development of hypoxia.

- Once supported ventilation is established, administer nondepolarising muscle relaxant.

- Start train of four (TOF) monitoring on NMT if available. 
- Sevoflurane $1-2 \%$ or supplemental doses of hyponotic drugs (e.g. propofol $0.5 \mathrm{mg} / \mathrm{kg}$ every $15-45 \mathrm{~s}$ ) must be administered during this time to maintain depth of anaesthesia.

- Continue to monitor vitals ( $\mathrm{HR}, \mathrm{SpO}_{2}$, NIBP on 1 minute cycle time) and gas analysis (oxygen and agent analysis, $\mathrm{ETCO}_{2}$ monitoring).

\section{Intubation:}

- Once 'zero single twitch' is established or adequate time for muscle relaxant to take effect has passed (1-3 minutes depending on which drug and dose is used), intubation can be performed.

- Gentle external laryngeal pressure with a BURP (backward, upward and rightward pressure) manoeuvre may be done by an experienced assistant to facilitate intubation.

- Confirm adequate ventilation (tidal volumes) and $\mathrm{ETCO}_{2}$.

- Confirm correct ETT placement clinically and secure the ETT at the appropriate level.

- Check cuff pressure with a manometer if available (keep cuff pressures $20-25 \mathrm{cmH}_{2} \mathrm{O}$ )

- If no manometer available, deflate the cuff gently and increase PIP to $20 \mathrm{cmH}_{2} \mathrm{O}$ - you should have a leak around the ETT

- Inflate the cuff with $0.5-1 \mathrm{ml}$ of air gently until the leak is no longer audible at PIP of $20 \mathrm{cmH}_{2} \mathrm{O}$

- If no leak is audible and ventilation is adequate without cuff inflation, do not inflate the cuff

- Remember to check ETT position and adequacy of ventilation after any re-positioning of the patient during the procedure.

\section{Emergence:}

- Muscle relaxant should be fully reversed

- Monitor with NMT if available

- Suctioning of a large bore NGT should be performed prior to extubation.

- Adequate suctioning of oro-pharynx must be performed.

- Extubate patient fully awake.

- Assess for adequate airway maintenance post-extubation

- Good air movement felt and heard around nose and mouth
- Normal movement of chest wall with no signs of obstruction

- Monitor for and rule out post-extubation airway or respiratory complications post-op in recovery:

- Post-extubation stridor

- Upper airway obstruction from residual effects of anaesthetic drugs

- Inadequate respiratory effort from residual neuromuscular blockade leading to hypoxia and hypercarbia

\section{References}

1. Kelly CJ, Walker RW. Perioperative pulmonary aspiration is infrequent and low risk in paediatric anaesthetic practice. Pediatric Anesthesia 2015; 25: 36-43.

2. Walker RW. Pulmonary aspiration in paediatric anaesthesia practice in the United Kingdom: a prospective survey of specialist paediatric centres over a one year period. Paediatric Anaesthesia 2013; 23: 702-11

3. Warner MA. Perioperative pulmonary aspiration in infants and children. Anesthesiol 1999; 90: 66-71.

4. Warner MA, Warner ME, Webber JG. Clinical significance of pulmonary aspiration during the peri-operative period. Anesthesiol 1993; 78: 56-62

5. Kalinowski $\mathrm{CPH}$, Kirsch JR. Strategies for prophylaxis and treatment for aspiration. Best Pract Res Clin Anaesthesiol 2004; 18: 718-737

6. Kluger MT, Shor TG. Aspiration during anaesthesia: review of 133 cases from the Australian anesthetic incident monitoring study (AIMS). Anaesthesia 1999; 54: 19-26

7. Engelhardt T. Rapid sequence induction has no use in pediatric anesthesia Pediatric Anesthesia 2015; 25: 5-8.

8. Gencorelli FJ, Fields RG. Complications during rapid sequence induction of general anesthesia in children: a benchmark study. Pediatric Anesthesia 2010; 20: 421-424.

9. Patel R, Lenczyk M, Hannallah RS. Age and the onset of desaturation in apnoeic children. Can J Anaesth 1994; 41: 771-4.

10. Spencer AO, Walker AM. Ultrasound assessment of gastric volume in fasted pediatric patients undergoing upper gastrointestinal endoscopy: development of a predictive model using endoscopically suctioned volumes. Pediatric Anesthesia 2014; 25: 301-308.

11. Scrimgeour GE, Leather NW. Gas induction for pyloromyotomy. Pediatric Anesthesia 2015; On-line publication 23 February

12. Rawicz M, Brandom B, Wolf $A$. The place of suxamethonium in pediatric anesthesia. Pediatric Anesthesia 2009; 19: 561-570

13. Neuhaus D, Schmitz A. Controlled rapid sequence induction and intubation - an analysis of 1001 children. Pediatric Anesthesia 2013; 23: 734-740.

14. Tobias JD. Pediatric airway anatomy may not be what we thought: implications for clinical practice and the use of cuffed endotracheal tubes. Pediatric Anesthesia 2015; 25: 9-19 